\title{
Critical evaluation of approaches toward mass deacidification of paper by dispersed particles
}

\author{
Antje Potthast $\mathbb{D} \cdot$ Kyujin Ahn
}

Received: 15 March 2016/ Accepted: 25 October 2016/Published online: 14 November 2016

(C) The Author(s) 2016. This article is published with open access at Springerlink.com

\begin{abstract}
Mass deacidification has been an important topic in cellulose science and will continue to be a critical issue as long as acidic books and paper-based materials are-a often major-part of library and archive stocks. Different means are available to judge the result of a deacidification treatment and to address its sustainability and efficacy. The present study compares deacidification by dispersed particles with procedures that apply homogeneously dissolved alkaline compounds, both under humid and dry accelerated aging conditions. Analysis by size-exclusion chromatography coupled to light scattering detection is used in combination with accelerated aging. The number of chain scissions, i.e. cellulose degradation, is the parameter used for evaluation, expressed as stabilization factors relative to the non-treated specimen. Upon deacidification with homogeneous solutions stability factors of about four were reached, while deacidification with dispersed particles gave only two times longer life times (stability factor of two). Mechanistic aspects are discussed in terms of alkaline reserve, cellulose degradation and mobilities of deacidification agents.
\end{abstract}

A. Potthast $(\square) \cdot$ K. Ahn

Christian-Doppler-Laboratory "Advanced Cellulose Chemistry and Analytics", Department of Chemistry, University of Natural Resources and Life Sciences, Konrad-Lorenz-Str. 24, 3430 Tulln, Austria

e-mail: antje.potthast@boku.ac.at
Keywords Mass deacidification - Cellulose · Stabilization · Accelerated aging - Suspension · Solution · Paper · Book

\section{Introduction}

Mass deacidification of archival and library material continues to be an important topic in conservation and preservation. Several tens of tons of books and archival material are treated every year worldwide. Goals of such treatments are clearly defined, either by general incentives-preserving valuable cultural assets for future generations and maintaining usability and readability of the printed matter-or by more scientific tasks: increasing the overall lifetime of the supporting paper matrix by neutralizing acids already present in the paper and by adding an alkaline reserve to ensure trapping of acids that might form later on during natural aging.

Different mass deacidification processes have been used to introduce the needed alkaline reserve into the treated books and archival materials. Those approaches can be distinguished by the way the alkaline substance is supplied. Large-scale operations apply the deacidification agent either as a solution or a suspension (dispersion) in non-aqueous, largely inert solvents, for a review on mass deacidification processes, please refer to Baty et al. (2010) and Blüher and Vogelsanger (2001). While dispersion methods, such as the Bookkeeper process, have dominated the 
market outside Europe, the ZfB2 process recently introduced in Germany brought the attention to dispersion methods back also here, as the process is claimed to be superior from an economic point of view (ZfB 2016). To evaluate Bookkeeper, the deacidification process chosen for instance by the US Library of Congress, a large study was conducted at the beginning of the 1990s (Buchanan et al. 1994). The ZfB2 process has been introduced into the market only a few years ago, but so far no performance data are available from independent literature and no benchmarking against other commercially applied processes has been published yet.

While the mechanism of the actual deacidification step is a straightforward chemical neutralization, a number of other questions remain regarding all mass deacidification treatments currently applied on a larger scale. Whitmore (1994) addressed some crucial issues in 1994, and their importance still prevails today: What factors determine how (and how fast) the primary agent forms the alkaline components, and how fast will the latter, in turn, react with the acids to be neutralized? Will that process be faster than the degradative action of the acid on the paper? How fast does the actual neutralization process proceed, and what are the preferred conditions for that? Are there additional benefits if the neutralization process is followed by exposure to controlled humidity and moderately elevated temperature, generally referred to as ,reconditioning “?

Acidic compounds are usually not homogeneously distributed in books or archival materials due to their origin. Acids, inorganic $\left(\mathrm{H}_{2} \mathrm{SO}_{4}\right)$ or organic (e.g. formic and acetic), are either originating from the papermaking process or a result of natural aging. The deacidification process has to reach all of them in the optimum case: acids, free or bound to paper (cellulose), by ionic interactions inside the matrix and the fiber or on the surface, inorganic or organic in nature. Once the reactive agent has met the acid, neutralization can take place. While the actual neutralization reaction, i.e. recombination of a hydronium cation and an hydroxyl anion to water, is very fast (in fact one of the fastest chemical elementary reactions known), the neutralization process as a whole may not happen immediately, as it involves mobility and accessibility issues in addition. It depends on the respective deacidification reagents in the mostly non-aqueous solvents currently applied in large-scale operations, the formation of the actual alkaline reagents from the primary reagents, and the mobility in the paper matrix, which determines the time before alkaline compound and acid are able to "meet". The timeline of the neutralization varies; it is process-specific and may even extend to several weeks after reconditioning of the material. On the long run a slow reacidification of the library material has been shown after natural aging (Höing 2008). When it comes to efficacy and performance of deacidification, the distribution of alkaline compounds throughout the book paper matrix has been proven to be highly important (Whitmore and Bogaard 1994; Ahn et al. 2012a). Previous investigation showed that even large amounts of alkaline agents were insufficient to significantly slow down acidinduced degradation reactions after accelerated aging if they are situated only on the paper surface (Ahn et al. 2012c). So far no methods exist that allow for a reliable quantification of deacidification reagents or alkaline reserve over the papers' cross-section, which is the most critical dimension. Visualization of the distribution can be approached by scanning electron microscopy coupled to energy-dispersive X-ray spectroscopy (SEM-EDX) of the respective surfaces and cross-sections, but this allows mainly judging the particle size and distribution on a qualitative level. It does not directly correlate with alkalinity or mobilities of ions or reagents. In addition, the preparation of the paper cross-section for SEM-EDX is tedious, timeconsuming and has a low sample throughput, and thus it is not suitable as routine analysis. Hence, only indirect methods have been applied to judge the deacidification performance: comparing the aging behavior of deacidified and non-deacidified samples, based either on physical test methods or chemical analysis of cellulose parameters, such as degree of polymerization (DP). The DP is not only directly related to cellulose chain integrity and thus reflects cellulose degradation immediately, it is also more direct than physical tests which depend on more influencing factors than cellulose chain degradation and in many cases are rather insensitive to DP changes.

Despite of several inherent drawbacks, artificial aging test has been accepted as the method of choice to analyze the efficacy of the deacidification process. Mass deacidification aims at long-term issues, but as these can evidently not be evaluated directly in the lab by observation of natural aging processes, artificial 
aging — in some cases also called accelerated aging is the only way out. The approach relies on several assumptions, the critical one being that accelerated aging will reproduce natural aging correctly not only with regard to material properties, but also with regard to molecular changes. Still, accelerated aging is the best (and only logical) test option available. During artificial aging in a humid atmosphere, hydrolytic cleavage of the cellulose molecules is triggered and considered to be the dominant process of paper degradation (Whitmore and Bogaard 1994). As expected for an acid-catalyzed process, the rate of the underlying reactions rises significantly in the presence of protons and is slowed in the presence of alkaline substances as the latter neutralize not only acids present but also those being formed de novo during aging. Analysis before and after this artificial aging allows conclusions about the efficiency of the overall treatment process (Buchanan et al. 1994; Banik 2005; Porck 2006; Andres et al. 2008; Ahn et al. 2013).

Artificial aging, independent of the protocol chosen, involves elevated temperatures and relative humidities at or above 50\%. These conditions favor hydrolytic damage of cellulose, which is mandatory for demonstrating the effect of deacidification in general. It is not clear, however, to what extent the elevated temperatures and the humidity induce a migration of the ions of the deacidification agent in the paper matrix and to what extent this migration is enhanced under conditions of accelerated aging. If the alkaline substance is evenly distributed around and within the cellulose fibers, further mobility would not change the aging result. On the other hand, if uneven spreading of the alkali component represents the initial situation, an induced movement under accelerated aging would pretend better results under test conditions than in reality. This would distort comparison between different deacidification methods. So far, no data are available on the influence of humidity during artificial aging after mass deacidification treatments.

The aim of the present investigation was to subject different book papers from real-world books-deacidified by a dispersion method that is now widely applied in practice - to humid artificial aging and to compare the results to deacidification methods that apply the alkaline precursor compound in solution. In addition, dry aging is applied for comparison, which largely restricts the ion mobility and "freezes" the alkaline reserve were it was initially deposited. Comparison of the two deacidification variants is expected to provide further insights into the topic of how to deposit alkaline reserves effectively.

\section{Materials and methods}

\section{Book samples}

The samples comprised 20 pairs of two identical books, published between 1910 and 1986 covering very different kinds of paper. One of the two books remained untreated (non-deacidified), the other was deacidified. One page in the middle of each book block was used. The samples used for deacidification in solution (Papersave process, ZfB) had already been part of a previous study on mass deacidification sustainability (Ahn et al. 2012a). All books exhibited an acidic surface (TAPPI 529 om.99 2004) before deacidification and a $\mathrm{pH}$ of $>6.5$ after deacidification. Before analysis and accelerated aging the books were stored identically at ambient conditions.

\section{Deacidification}

All deacidified books were part of commercial deacidification runs and were supplied by libraries. Deacidification by the dispersion method was accomplished by $\mathrm{CaCO}_{3} / \mathrm{MgO}$ in heptane (ZfB2, Germany). Book samples deacidified by homogeneously dissolved chemicals used magnesium ethoxide in combination with titanium alkoxide (ethoxide and isopropoxide) in hexamethyldisiloxane (HMDS) (Papersave Process, ZfB, Germany).

\section{Aging}

Each sample page was bisected horizontally. The upper half of the page was left as a non-aged control, and the lower half was aged. Accelerated aging conditions were $80{ }^{\circ} \mathrm{C}$ and $65 \%$ relative humidity (RH) for 40 days, based on ISO 5630-3:1996, using a Q-Sun Xe-3 tester (Q-LAB, USA). Samples were stacked between neutral blotting papers (KLUG Conservation $\mathrm{GmbH}$, Germany). All papers, including a Whatman no. 1 paper, were aged under exactly similar conditions at the same time. Dry aging was performed at $105{ }^{\circ} \mathrm{C}$ for 100 days in a drying oven. 
Whatman paper served as an internal reference material for aging; it was not deacidified. For dry aging, time and temperature were adjusted in a way that the number of chain scissions was similar to the humid aging variant for Whatman paper, thus reflecting about the same aging severity. For Whatman paper, aging resulted in a chain scission number of 0.9 under humid conditions, compared to 0.87 under dry conditions.

Sample preparation for gel permeation chromatography (GPC) and GPC conditions

Approximately $100 \mathrm{mg}$ (dry) of each sample from various areas (top, bottom, center) of one whole page of the book was combined to minimize possible sample inhomogeneity and was disintegrated in water. $50 \mathrm{mg}$ of the drained, water-wet samples was dissolved in DMAc/LiCl 9\% (weight [w]/volume [v]), based on Potthast et al. (2015). Cellulose analysis was performed on a GPC-MALLS system which yields the molecular weight distribution (MWD) and the derived statistical molecular weight parameters.

The GPC system consisted of a MALLS detector (Wyatt Dawn DSP; Wyatt Inc., Santa Barbara, CA, USA) with an argon ion laser $\left(\lambda_{0}=488 \mathrm{~nm}\right)$ and a refractive index detector (Shodex RI-71, Japan), degasser (Dionex DG-2410), autosampler (HP 1100), pulse damper pump (Kontron pump 420), and column oven (Gynkotek STH 585). Four serial GPC columns, PLgel-mixed ALS, $20 \mu \mathrm{m}, 7.5 \times 300 \mathrm{~mm}$ (Agilent, Waldbronn, Germany) were used as the stationary phase. Operating conditions: $1.00 \mathrm{~mL} / \mathrm{min}$ flow rate, $100 \mu \mathrm{L}$ injection volume, and $45 \mathrm{~min}$ run time. $\mathrm{DMAc} / \mathrm{LiCl}(0.9 \%, \mathrm{w} / \mathrm{v})$, after filtering through a $0.02 \mu \mathrm{m}$ filter, was used as the eluent. Data were evaluated with Astra and GRAMS/32 software.

The number of chain scissions (NCS) was calculated based on the degree of polymerization before $\left(\mathrm{DP}_{\mathrm{o}}\right)$ and after (DP) accelerated aging, using the following equation:

$N C S=\frac{D P o}{D P}-1$

DPo and DP are based on the weight-average molar mass obtained as an absolute value from SECMALLS (Hon 1985; Johansson et al. 2000; Bouchard et al. 2006; Calvini and Gorassini 2006).
The stability factors (SF) are calculated based on average chain scissions during accelerated aging without and with deacidification treatment.

$\mathrm{SF}=\mathrm{NCS}($ not deacidified)/NCS (deacidified)

\section{Statistical analysis}

Treatment effects were analyzed statistically with linear mixed models (Pinheiro and Bates 2000) in the R statistical environment (Pinheiro et al. 2016) using the contributed package "nlme". A restricted maximum likelihood estimation of fixed (deacidification method) and random (book identity) effects was performed for the relative difference of chain scissions between non-acidified and acidified paper as the response variable by function lme (nlme). Nonparametrical tests (Mann-Whitney-U-test) were performed as well.

\section{Results and Discussion}

The samples used for this study were original books to simulate the real-world situation in libraries and deacidification treatments as closely as possible. Hence, we neither knew the natural aging history of the books nor under which particular conditions they had been stored in the past. We applied the "identical book"-method in which a pair of the same book (same edition) is evaluated in its deacidified and in its nondeacidified form after accelerated aging. This approach reflects the reality of deacidification appropriately because it covers not only the mere acidity of the paper, but also different morphologies and surfaces.

The use of original books might be seen as a drawback which results in higher variation of the data (which we tried to compensate by a larger sample number). Still, original samples evidently reflect the real-world situation in libraries better than model papers. In a previous study (Ahn et al. 2012a), this approach has been found to be quite suitable to study mass deacidification, since this method readily revealed differences between different deacidification variants. The two identical book copies showed, in almost all cases, a similar molar mass distribution before accelerated aging, even if natural aging (i.e. the age of the book) exceeded 50 years. In the present 
study, the samples were also compared in untreated and treated form before artificial aging.

Whether a mass deacidification treatment has been successful or not depends on a number of different parameters. From the molecular point of view, the protection of cellulose integrity is the most important issue as it eventually translates into material properties and ensures flexibility of the paper and makes sure that further handling has no negative impact. It is directly related to mechanical properties of the entire paper matrix. While a large set of accelerated aging data is available for deacidification by homogeneous reagent solutions (e.g. Papersave, Booksaver), there are no comparable data available for the novel dispersion method $\left(\mathrm{CaCO}_{3} / \mathrm{MgO}\right.$ in heptane) as this procedure had not been applied on a large scale until very recently. The data presented here are a first treatise of such dispersion methods, applying similar evaluation methods as used previously for deacidification with homogeneous reagents (Ahn et al. 2011, 2012a), but it is obviously not meant as a full-scale sustainability study of currently applied mass deacidification techniques. We discuss the comparison of the methods in view of the behavior of alkaline reserve deposited by dispersion methods, and its transport into the paper matrix during aging.

In order to visualize whether a deacidification treatment had the desired effect, we first analyzed the sample papers before and after mass deacidification without accelerated aging. The average molar mass was $296 \mathrm{~kg} \mathrm{~mol}^{-1}$ ( $=\mathrm{DP}$ 1827) for the non-deacidified papers and $269 \mathrm{~kg} \mathrm{~mol}^{-1}$ ( $\hat{=} \mathrm{DP}$ 1660) for the deacidified papers. Overall, a slight degradation after mass deacidification, relative to the non-deacidified copy, was apparent for all samples, although the change was statistically not significant (analysis of variance, ANOVA test). The alkaline medium did not have a strong effect with regard to $\beta$-elimination reactions as has been already demonstrated for other mass deacidification methods (Ahn et al. 2013). The overall variation was higher for the dispersion method.

Figure 1 illustrates a selection of different book samples treated with a dispersion method showing different outcomes of deacidification. Samples given in Fig. 1a, c, d (\#10, \#17, and \#5) showed no degradation effect after deacidification prior to aging, sample b (\#1) did.

More interesting than the changes occurring directly upon deacidification treatment is the question how the samples behave after aging according to different accelerated aging protocols. To evaluate the performance, we calculated the number of chain scissions relative to the material before deacidification. This is a suitable measure because it is largely independent of the initial molar mass, which is of course different for each sample, and allows evaluation and comparison despite different molar mass values of the individual specimens. The lower the number of chain scissions after accelerated aging, the better the paper has been protected by the deacidification treatment.

Dry aging needed a significantly longer time to reach a similar level of degradation. Whether dry aging and humid aging yield overall similar results on the molecular level is still a matter of debate. Whitmore and Bogaard (1994) showed no large difference in the formation of carbonyls and carboxyl groups, whereas Erhardt and Mecklenburg (1995) argued that both techniques produce different degradation products. However, this aspect is not critical for the present issue as we compared data within similar systems and considered cellulose integrity (DP) as the main parameter.

\section{Stability factor}

The stability factor can be used as a quite practical number to compare stabilization treatments. It estimates how many times longer a paper would last after a respective treatment relative to a non-treated sample. The factor is based on the number of chain scissions having occurred during accelerated aging. Figure 2 shows the data for chain scissions used for calculating the stabilization factor. With decreasing numbers of chain scissions, the benefit on stability increases. The non-deacidified set of papers used in the dispersion method showed an average of a 0.85 chain scissions (median 0.76) after humid aging, which was lowered by deacidification to 0.40 (median 0.40 ). Hence, the paper aged about 2 times slower compared to the nondeacidified variant. Data so far available for a dispersion process are from BookKeeper which operates with magnesium oxide particles in an organic solvent, in which a stability increase of 2-4 times is a desired value, the Library of Congress requiring a factor of 3 (Buchanan et al. 1994).

The papers used in homogeneous deacidification had 0.61 (median 0.60) scissions in untreated form, which was lowered to average 0.14 scissions (median 

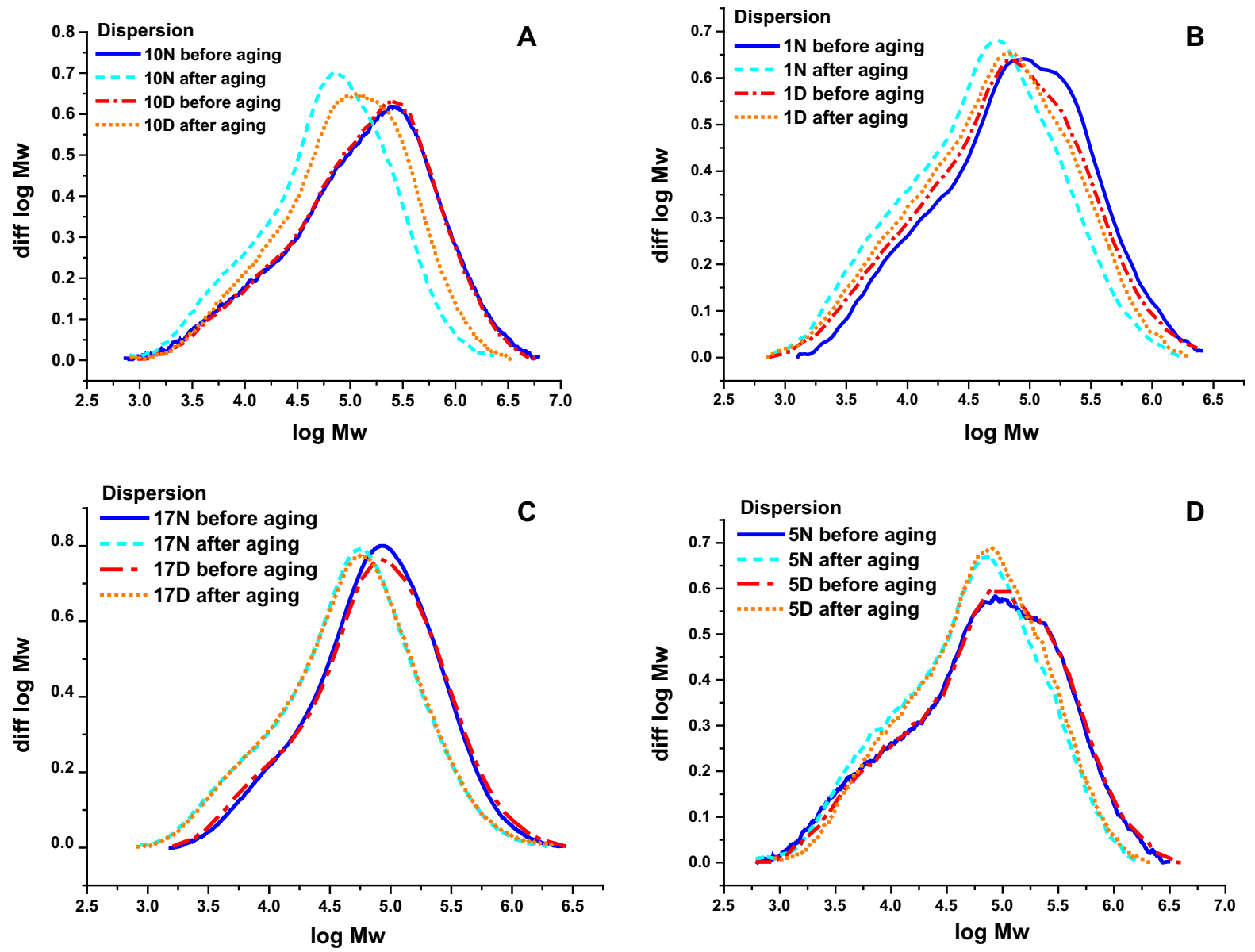

Fig. 1 Molar mass distributions of papers from four different book sets (samples a-d) before and after deacidification, and before and after aging. $\mathrm{N}$ (after the sample name): non-deacidified; D (after the sample name): deacidified

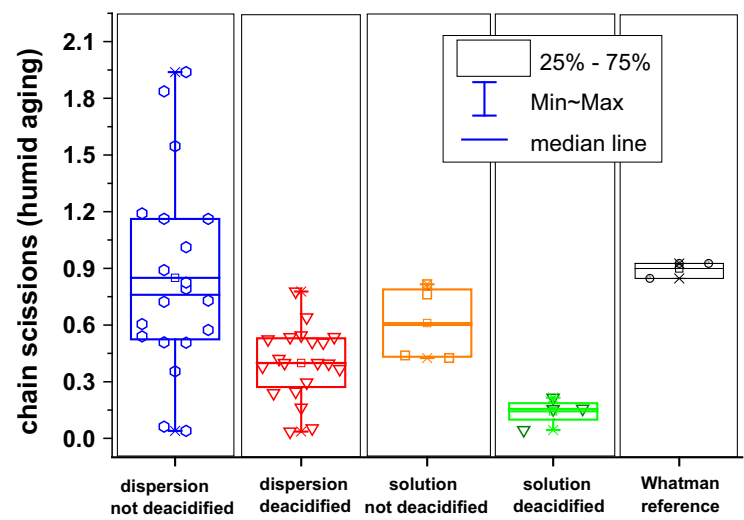

Fig. 2 Chain scissions after accelerated aging under humid conditions, for book samples deacidified by the dispersion method $(\mathrm{N}=20)$ and the solution procedure $(\mathrm{N}=4)$
0.16) after deacidification, resulting in a stabilization factor of about 4 . This is in agreement with previous studies on the Papersave process (homogeneous deacidification) based on mechanical tests which measured a factor of 3-4 in dependence on the deacidification technique applied (Liers 2001; Balazic et al. 2007) and is also in line with a report by Strlic et al. (2005). Also we have found similar values for dissolution treatments previously (Ahn et al. 2012b, c). The individual values are mainly influenced by differences in the book paper per se, but also by the samples' behavior in the analytical method (different solubility and dissolution behavior upon SEC-MALLS). While the variance of chain scission before deacidification is large, it becomes more narrow after deacidification 
(Fig. 2). The overall number of books for the solution deacidification was lower than for the dispersion treatment as the former samples (homogeneous process) has alreay been part of a comprehensively analyzed larger set for this type of deacidification (Ahn et al. 2012a, c). Overall, the differences found in this study, by subjecting the differently deacidified book papers to the same aging procedure, were statistically significant, with the solution procedure extending the life time of the material about two times longer than the dispersion variant.

Reasons for the different behavior observed are the penetration of the alkaline reserve into the paper and its deposition upon and within the fiber web, which is often a bottle neck: sometimes significant amounts of the active agents are left at the paper surface due to limited penetration caused by surface morphology of the paper that is a result of, for instance, coating and calandering.

When a dispersion is used for deacidification, the size of the dispersed particles is an essential factor, even though a recent study demonstrated that reaching the whole cross-section of a paper sheet can also be accomplished in a heterogeneous dispersion system (ZfB 2016). So what is the actual reason for differences in performance between dispersion and solution treatments? The critical issue is not just penetration into the macroscopic pore system of the paper matrix, it is penetration into the cellulosic fibers themselves. The pore size here is, however, an order of magnitude (or even more) smaller compared to pores within the paper sheet. Any deacidification reagent has to penetrate the larger-pore paper web as well as the smallpore cellulose fibers in order to protect the cellulose on a quasi-molecular level within the cellulose fibrils.

The average pore size in the macroscopic paper web is roughly between 1 and $10 \mu \mathrm{m}$ (Resch et al. 2010; Bennis et al. 2010), the average pore size in pulp fibers is around 1-100 nm with mainly mesopores and macropores (Andreasson et al. 2003; Aarne et al. 2012; Lovikka et al. 2016). Suspended particles have to be small enough not only for penetrating the paper matrix, but also for invading individual pulp fibers. While the $\mathrm{ZfB} 2$ process appeared well suited to deposit an alkaline reserve over the entire surface of the sheet (Fig. 3, right), its ability to penetrate into the pulp fiber is limited (see ZfB 2016). This is clearly visible at cross-sections of papers deacidified by dispersion systems: the deposition of the alkaline reserve is quite homogeneous on the paper matrix scale, the reagent filling interstices, pores and interfiber spaces. It is much less pronounced within the fibers, which is simply due to the particle size of the deacidification reagent, limiting access to the fiber interior. We can reasonably assume, therefore, that the differences seen in the aging study under humid conditions reflects the limited penetration of the active agent into the actual pulp fibrils, which is less problematic for a homogeneous deacidification solution (Ahn et al. 2012a). The paper surface analysis by SEM-EDX before and after treatment by dispersions is shown in Fig. 3. Although the different accessibilities of (nano)particles versus solutions might be considered expectable, the present study shows the effect at the molecular level and provides a direct comparison of solution and dispersion deacidification.

The used particles in the dispersion deacidification are calcium carbonate and magnesium oxide. Nevertheless, the distributions of magnesium and calcium at the papers' surface are somewhat different, as shown in Fig. 4. More magnesium was detected along the edge of the fiber while more calcium was found in the corner of a dented area as highlighted in Fig. 4. Whether this points to a disintegration of the initial particles resulting in secondary species with different deposition behavior cannot be answered at present. Apart from different effects on the $\mathrm{pH}$ after reconditioning of the paper, to date we have no information on the difference in deacidification behavior between different species $(\mathrm{Ca}, \mathrm{Mg})$.

Dry aging

The dry aging protocol in our study was used as a means to observe the papers with "frozen" distribution of the alkaline reserve because mobility becomes rather limited under those conditions of limited humidity. Dry aging would thus suppress a possible benefit of classical (humid) aging approaches. Dry aging emphasizes the importance of a uniform and homogenous distribution of the alkaline reserve since later averaging mobilities of ions that occur under humid conditions are now largely prevented. Although the data for the dry aging have a high variance, this trend was visible. For both deacidification systems, solution and dispersion, the low humidity largely suppressed the protective effect of the alkaline reserve (Fig. 5). The dispersion variant showed, also due to 

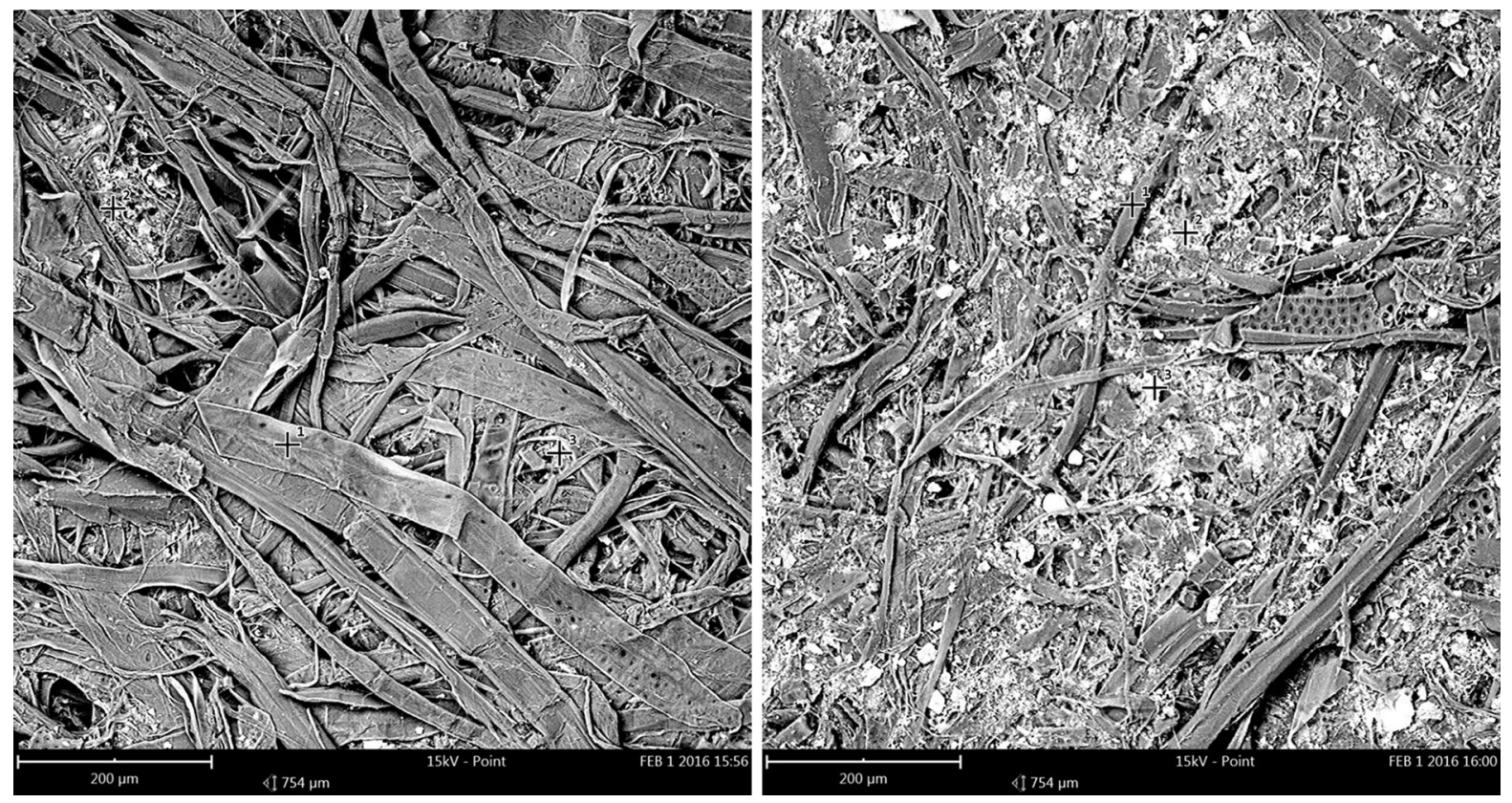

Fig. 3 SEM of a book papers. Left without deacidification treatment; right deacidified by a dispersion method
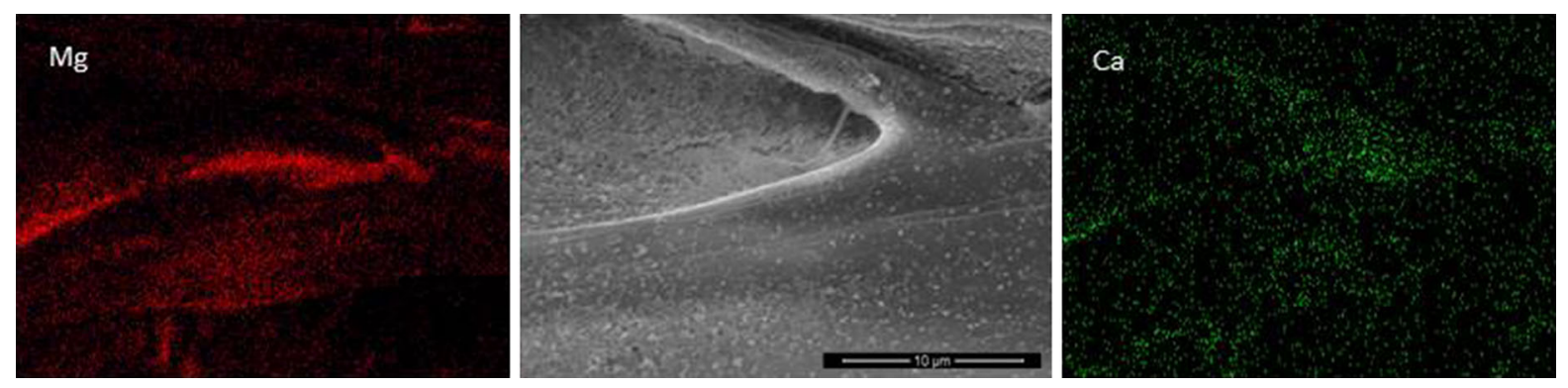

Fig. 4 SEM-EDX of a book paper surface after deacidification according to the dispersion method. Left Distribution of magnesium species; right distribution of calcium species; middle SEM of this area

the large variation in the book pages, no significant effect (median), while the solution procedure still afforded an—albeit very small—benefit (Fig. 5).

Chain cleavage is triggered by local hydrolysis of glycosidic bonds, it cannot be prevented through neutralization if the counteragents cannot approach the site of acidity or, vice versa, if volatile acids (formic acid, acetic acid) fail to encounter the alkaline reserve. These two ways exist for the alkaline pool and the acidic compounds to meet. Relocating the ions of the alkaline substance per se requires a mobile aqueous phase. This mechanism of neutralization is a "relay" of hydronium and hydroxyl ions, commonly referred to as Grotthuss mechanism (de Grotthuss
1806), in a way that protons and hydroxyl ions are transmitted through an aqueous phase by a chain mechanism, which allows fast proton and hydroxyl ion transport and accounts for the fact that the actual site of neutralization (proton/hydroxyl ion recombination) can be spatially different from the actual acidic group. The ions of the alkaline reserve under dry conditions are spatially confined and neither able to diffuse to the site of acidity nor to neutralize it according to the Grotthuss conductive mechanism (Agmon 1995; Cukierman 2006; Markovitch and Agmon 2007). The second way of neutralization is by trapping volatile acids (e.g. formic and acetic acid) by the immobile alkaline reserve at the point where it is 


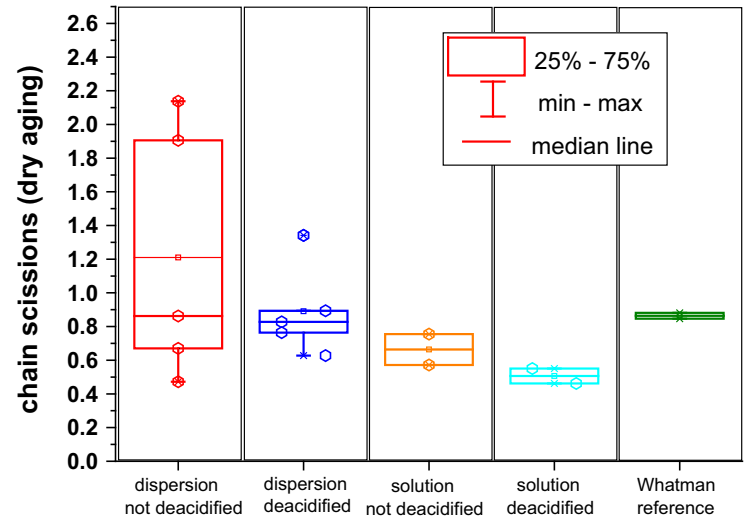

Fig. 5 Chain scissions after accelerated aging under dry conditions for book paper samples deacidified by dispersion $(\mathrm{N}=5)$ and solution procedures $(\mathrm{N}=2)$

deposited. Formation of such acidic volatiles from paper upon aging has been intensively studied and confirmed (Cincinelli et al. 2016; Becker et al. 2016).

\section{Conclusions}

The direct comparison between deacidification processes applied on a commercial scale showed significant differences between dispersion and dissolution variants upon standard humid aging, according to stability factors based on the number of chain scissions, with the homogeneous variant being superior. The better performance of deacidification in homogeneous solution, about twice as good in units of the stability factor, can be explained by the better penetration of both the macroscopic paper matrix with its large pores and voids-which also dispersion methods are able to reach-and the cellulose fibers with its much smaller pores, which homogenous reagent solutions can enter, but dispersed particles fail to access due to their sheer size. Dry (hot) aging further supported the significance of a uniform penetration of the alkaline reserve into the fiber matrix since ion mobility due to humidity is excluded in this case and neutralization can rely only on the direct encounter of the antipodes.

Acknowledgments The authors are grateful to Dr. A. Tischer, Westfälische Wilhelms-Universität Münster, for statistical data evaluation. Open access funding provided by University of Natural Resources and Life Sciences Vienna (BOKU).
Open Access This article is distributed under the terms of the Creative Commons Attribution 4.0 International License (http:// creativecommons.org/licenses/by/4.0/), which permits unrestricted use, distribution, and reproduction in any medium, provided you give appropriate credit to the original author(s) and the source, provide a link to the Creative Commons license, and indicate if changes were made.

\section{References}

Aarne N, Kontturi E, Laine J (2012) Influence of adsorbed polyelectrolytes on pore size distribution of a water-swollen biomaterial. Soft Matter 8:4740-4749

Agmon N (1995) The Grotthuss mechanism. Chem Phys Lett 244(5-6):456-462

Ahn K, Henniges U, Blüher A, Banik G, Potthast A (2011) Sustainability of mass deacidification. Part 1: concept, selection of sample books, $\mathrm{pH}$-determination. Restaurator 32:193-222

Ahn K, Banik G, Potthast A (2012a) Sustainability of massdeacidification. Part 2: evaluation of alkaline reserve. Restaurator 33(1):48-75

Ahn K, Henniges U, Banik G, Potthast A (2012b) Is cellulose degradation due to $\beta$-elimination processes a threat in mass deacidification of library books? Cellulose 19(4): $1149-1159$

Ahn K, Henniges U, Banik G, Potthast A (2012c) Nachhaltigkeit in der Massenentsäuerung von Bibliotheksgut. In: Altenhöner R, Blüher A, Mälck A, Niggemann E, Potthast A, Schneider-Kempf B (Hrsg) Eine Zukunft für saures Papier. Konservierung und Restaurierung. Zeitschrift für Bibliothekswesen und Bibliographie. Sonderband 106. Vittorio Klostermann, Frankfurt am Main

Ahn K, Rosenau T, Potthast A (2013) The influence of alkaline reserve on the aging behavior of book papers. Cellulose 20(4):1989-2001

Andreasson B, Forsström J, Wagberg L (2003) The porous structure of pulp fibres with different yields and its influence on paper strength. Cellulose 10:11-123

Andres H, Blüher A, Grossenbacher G, Reist M, Vogelsanger B, Wälchli M (2008) The papersave Swiss process: quality control and efficacy. Restaurator 28:3-28

Balazic A, Habicht S, Smodis M, Kolar J, Strlic M (2007) Extending the useful life of paper-evaluation of the effect of various preservation actions. In: Conference proceedings-museum microclimates 2007. The National Museum of Denmark, Copenhagen, pp 39-41

Banik G (2005) Mass deacidification technology in Germany and its quality control. Restaurator 26:63-75

Baty JW, Maitland CL, Minter W, Hubbe MA, Jordan-Mowery SK (2010) Deacidification for the conservation and preservation of paper-based works: a review. BioResources 5(3):1955-2023

Becker M, Meyer F, Jeong M-J, Ahn K, Henniges U, Potthast A (2016) The museum in a test tube-adding a third dimension to the evaluation of the impact of volatile organic acids on paper. Polym Degrad Stab 130:109-117 
Bennis H, Benslimane R, Vicini S, Mairani A, Princi E (2010) Fibre width measurement and quantification of filler size distribution in paper-based materials by SEM and image analysis. J Electron Microsc 59(2):91-102

Blüher A, Vogelsanger B (2001) Mass deacidification of paper. Chimia 55(11):981-989

Bouchard J, Méthot M, Jordan B (2006) The effects of ionizing radiation on the cellulose of wood free paper. Cellulose 13:601-610

Buchanan S, Bennett W, Domach MM, Melnick SM, Tancin C, Whitmore PM, Harris KE, Shahani C (1994) An evaluation of the bookkeeper mass deacidification process. Technical Evaluation Team Report, For the Preservation Directorate, Library of Congress, Pittsburgh, PA, 22 September 1994

Calvini P, Gorassini A (2006) On the rate of paper degradation: lessons from the past. Restaurator 27(4):275-290

Cincinelli A, Martellini T, Amore A, Dei L, Marrazza G, Carretti E, Belosi F, Ravegnani F, Leva P (2016) Measurement of volatile organic compounds (VOCs) in libraries and archives in Florence (Italy). Sci Total Environ 572: 333-339

Cukierman S (2006) Et tu Grotthuss! Biochim Biophys Acta 1757(8):876-878

de Grotthuss CJT (1806) Sur la décomposition de l'eau et des corps qu'elle tient en dissolution à l'aide de l'électricité galvanique. Ann Chim 58:54-73

Erhardt D, Mecklenburg MF (1995) Accelerated VS natural aging: effect of aging conditions on the aging process of cellulose. In: Proceedings of materials research society symposium, vol 352, pp 247-270

Höing H (2008) Das Bückeburger Verfahren zur Massenentsäuerung von Archivgut. Archivar 61:163-166

Hon DNS (1985) Mechanochemistry of cellulosic materials. In: Kennedy JF, Phillips GO, Wedlock DJ, Williams PA (eds) Cellulose and its derivatives: chemistry, biochemistry and applications, chapter 6. Ellis Horwood Limited, Chichester, pp 71-86

Johansson EE, Lind J, Ljunggren S (2000) Aspects of the chemistry of cellulose degradation and the effect of ethylene glycol during ozone delignification of Kraft pulps. J Pulp Pap Sci 26(7):239-244

Liers J (2001) Mass deacidification: the efficacy of the papersave process. PapierRestaurierung: Mitteilungen der IADA 2(Suppl):57-62
Lovikka VA, Khanjani P, Väisänen S, Vuorinen T, Maloney TC (2016) Porosity of wood pulp fibers in the wet and highly open dry state. Microporous Mesoporous Mater 234:326-335

Markovitch O, Agmon N (2007) Structure and energetics of the hydronium hydration shells. J Phys Chem A 111(12): 2253-2256

Pinheiro JC, Bates DM (2000) Mixed-effects models in S and S-Plus. Springer, New York. doi:10.1007/978-1-44190318-1

Pinheiro J, Bates D, DebRoy S, Sarkar D, R Core Team (2016) nlme: linear and nonlinear mixed effects models

Porck HJ (2006) The Bookkeeper process and its application in the National Library of the Netherlands (KB). In: Blüher A, Grossenbacher G (eds) Save paper! Mass deacidificationtoday's experience-tomorrow's perspectives. Swiss National Library, Bern, pp 37-42

Potthast A, Radosta S, Saake B, Lebioda S, Heinze T, Henniges U, Isogai A, Koschella A, Kosma P, Rosenau T, Schiehser S, Sixta H, Strlic M, Strobin G, Vorwerg W, Wetzel H (2015) Comparison testing of methods for gel permeation chromatography of cellulose: coming closer to a standard protocol. Cellulose 22(3):1591-1613

Resch P, Bauer W, Hirn U (2010) Calendering effects on coating pore structure and ink setting behavior. Tappi J 9(1):27-35

Strlič M, Kolar J, Malešič J, Kočar D, Šelih VD, Pihlar B, Haillant O, Pedersoli JL Jr, Scholten S, Rychlý J, Rychlá L, Fromageot D, Lemaire J (2005) Chapter 11: Stabilisation strategies. In: Strlič M, Kolar J (eds) AGEING and stabilisation of paper. National and University Library, Ljubljana, pp 181-198

TAPPI - Technical Association of the Pulp and Paper Industry (ed) (2004) Surface pH measurement of paper, TAPPI 529 om.99. In: TAPPI Test Methods. TAPPI PRESS, Atlanta

Whitmore PM (1994) Evaluation of the bookkeeper process chemistry. In: An evaluation of the bookkeeper mass deacidification process. Technical Evaluation Team Report, For the Preservation Directorate, Library of Congress, Pittsburgh, PA

Whitmore PM, Bogaard J (1994) Determination of the cellulose scission route in the hydrolytic and oxidative degradation of paper. Restaurator 15(1):26-45

ZfB2: Zentrum für Bucherhaltung Leipzig (2016) http://www. zfb.com/en/mass-deacidification/zfb2-method. Accessed 16 Aug 2016 